\title{
O ACOLHIMENTO AO PACIENTE ALCOOLISTA NOS SERVIÇOS DE URGÊNCIA E EMERGÊNCIA \\ - revisão integrativa
}

\author{
Salete Büll Pellegrini*, Jane Kelly Oliveira Friestino**, Denise Cuoghi de Carvalho Veríssimo Freitas*** \\ Autor para correspondência: Jane Kelly Oliveira-Friestino - jane.friestino@uffs.edu.br \\ * Enfermeira especialista em Dependência Química, Centro Universitário Salesiano de São Paulo, Campus Liceu \\ ** Enfermeira, doutora e mestre em Saúde Coletiva - Epidemiologia. Professora da pós-graduação no Centro Universitário \\ Salesiano de São Paulo, Campus Liceu e professora adjunta da Universidade Federal da Fronteira Sul, campus Chapecó-SC \\ *** Fisioterapeuta, doutora em Ciências da Saúde. Professora da pós-graduação no Centro Universitário Salesiano de São \\ Paulo, Campus Liceu
}

\begin{abstract}
Resumo
Os serviços de urgência e emergência recebem pacientes de alta complexidade e com diversas situações de risco de vida, onde muitos fatores podem influenciar na qualidade da assistência prestada aos usuários. Este estudo teve por objetivo revisar a literatura acerca da aplicação do conceito de Acolhimento e suas contribuições frente à demanda específica do indivíduo alcoolista nos serviços de urgência e emergência. Foi realizada uma revisão integrativa da literatura no período de 2004 a 2014, nas seguintes bases de dados Medline, Lilacs, Bdenf e Scielo, com base nos descritores: acolhimento, emergência e álcool. O total de artigos encontrados nas bases de dados foi de 137 estudos, dentre os quais 28 atenderam aos critérios de inclusão. Após a leitura, apenas og compuseram a amostra final dos artigos de acordo com a pergunta norteadora do estudo. Foram constatadas 02 categorias que apontaram a contribuição do Acolhimento ao indivíduo alcoolista nos serviços de urgência e emergência: conhecimento do perfil do usuário dos serviços de urgência e emergência e o Acolhimento e o papel dos profissionais. Conclui-se que a utilização das premissas do Acolhimento em Saúde no atendimento aos indivíduos alcoolistas, deve basear-se no reconhecimento das especificidades e necessidades próprias aos diferentes perfis de usuários e no delineamento e a clareza do Acolhimento, para que os profissionais entendam-se como agentes transformadores da realidade.
\end{abstract}

Palavras-chave: Acolhimento; Emergências; Alcoolismo; Saúde Mental. 


\title{
HUMANIZED INTAKE OF INCOMING ALCOHOLIC PATIENTS IN URGENCY AND EMERGENCY SERVICES \\ - integrative review •
}

\begin{abstract}
Emergency care services receive highly complex patients, and various life-threatening situations, and many factors can influence the quality of care provided to patients. This study reviewed the literature on the application of the concept of humanized intake and its contributions to the specific demand of alcoholic patients in urgency and emergency services. An integrative literature review of scientific reports on the theme from 2004 to 2014 was performed in the Medline, Lilacs, Scielo and BDENF databases, based on descriptors: humanized inbound patient intake, emergency care and alcoholism. A total of 137 studies was found in the databases, 28 of which met the inclusion criteria being thus selected for analysis. Out of those, og studies were selected. Two categories pointing to the institution's contribution to the individual alcoholic inbound patient primary care in urgency and emergency services could be identified: knowledge about the user profile in the emergency services and emergency and humanized patient intake and the role of professionals involved. It was identified that the implementation of humanization in health care should go beyond following protocol. It should seek integral humanized care to the alcoholic patient. It is concluded that the use of the premises of humanized intake in alcoholic patients, should be based on the recognition of the specific needs of the different user profiles and in the design and clarity of humanized inbound patient, for professionals to understand themselves as agents of change in reality.
\end{abstract}

Kepwords: User Embracement; Emergencies; Alcoholism; Mental Health.

\section{INTRODUÇÃO}

Desde a metade do século $X X$ a dependência química passou a ser discutida pela área da saúde, sendo considerados pacientes aqueles que consumiam de forma abusiva os diferentes tipos de substâncias. A assistência era ofertada de acordo com sinais, sintomas e transtornos mentais, que por vezes eram vinculados a condutas associadas a desvio de caráter. ${ }^{(1)}$

Dentre as substâncias, o álcool é considerado a droga mais consumida desde os relatos bíblicos, porém, o alcoolismo começou a ser tratado como doença na primeira metade do século XIX, com o intuito de promover tratamento a todos os indivíduos que apresentavam complicações por fazerem uso contínuo e abusivo de álcool, e que não recebiam nenhum tipo de atenção a saúde por estarem diretamente ligados à vergonha de carregar o estigma do abuso da substância. ${ }^{(2)}$

Em 1977, foi adotada pela Organização Mundial Da Saúde (OMS) a definição da dependência de álcool como uma "síndrome com contínuo de gravidade, tendo duas distinções claras: o uso e a dependência".(2) Desde então, essa síndrome é considerada como um problema que atinge cerca de 10 a $12 \%$ da população de todo o mundo e, fatores como gênero, etnia, idade, ocupação, grau de instrução e estado civil, podem estar relacionados 
ao uso abusivo de álcool, tendo como consequência a dependência. ${ }^{(3)}$

No Brasil, cerca de $11 \%$ da população masculina e $02 \%$ da feminina consomem álcool diariamente, não havendo consumo de álcool isento de riscos. Entre esses riscos podemos citar uma forma de consumo conhecida como binge (consumo excessivo esporádico), onde ocorre a ingestão de cinco ou mais de cinco doses de álcool para homens e quatro para mulheres em um mesmo período. ${ }^{(3)}$ Além disso, cerca de $13 \%$ da população de todo o país são dependentes de álcool, e estima-se que no estado de São Paulo a dependência atinja cerca de dois milhões de pessoas. ${ }^{(4)}$

Há muitos anos o consumo de álcool vem sendo de forma contínua e abusiva considerada a droga psicoativa com maior uso entre as populações de todo mundo, fato tão importante registrado nos últimos anos por causar inúmeros danos à saúde física e psicológica dos indivíduos, e ser avaliado como problema de saúde pública. ${ }^{(4)}$

O alcoolismo é reconhecido como um fator de fragilização da saúde da população em situação de rua, sendo pessoas excluídas das estruturas convencionais da sociedade, vivendo na linha da indigência ou pobreza absoluta. (5) Há um consenso da associação entre alcoolismo e população de rua, e frente a este panorama, há a ocorrência de grande suscetibilidade a outras enfermidades, que por vezes necessitam de cuidados em saúde nos diversos níveis de complexidade do Sistema Único de Saúde (SUS). ${ }^{(5)}$

Embora um grande contingente da população conviva como alcoolismo, ainda existe um estigma que permeia a entrada do indivíduo alcoolizado ou dependente de álcool nos serviços de urgência e emergência, gerando atitudes de desprezo e marginalização do doente. ${ }^{(6)}$

No âmbito do SUS, o Acolhimento apresenta-se como uma das diretrizes da Política Nacional de Humanização $(\mathrm{PNH})$ e tem como principal proposta descentralizar a abordagem médica, multiplicando as orientações de acordo com a deman$\mathrm{da}$, melhorando o ambiente e a escuta por parte dos profissionais em relação às expectativas da busca de resolução dos problemas de saúde da clientela. ${ }^{(7)}$

O Acolhimento em saúde no SUS não se trata apenas do uso de tecnologias e ciência na assistência com intuito da prevenção, promoção de doenças e seus agravos, o profissional compromete-se a promover e zelar pela vida do cidadão, estabelecendo uma relação de vínculo entre os indivíduos e seus familiares. ${ }^{(8)}$

No hospital a demanda e o fluxo intenso de atendimentos e procedimentos realizados muitas vezes dispõem de excelentes recursos para resolução dos problemas de saúde dos usuários destes serviços, mas pouco se ouve ou se conhece dos medos e angústias causadas pelo enfrentamento da expectativa da resolução ou internação em muitos casos. O Acolhimento nos hospitais não é somente voltado para a educação e treinamento dos profissionais, mas sim, para promoção de mudanças na estrutura, nos processos de trabalho, na produção de saúde da população, tornando o momento menos traumático. ${ }^{(9)}$

Nesse contexto, os serviços de urgências e emergência recebem pacientes de alta complexidade, $e$ com diversas situações de risco iminente de vida, onde muitos fatores podem influenciar na qualidade da assistência prestada aos usuários deste serviço. ${ }^{(10)}$ Muitos destes pacientes são alcoolistas, e necessitam de atendimento, pois o uso e o abuso de álcool estão diretamente relacionados a problemas de agravos a saúde, socioculturais e econômicos, podendo apresentar maior risco de acidentes automobilístico, violências, comportamento de risco ligado ao sexo sem proteção, uso de drogas psicotrópicas, intoxicação alcóolica, síndrome da abstinência alcóolica, transtornos psiquiátricos ou ainda condições clínicas como hipertensão, doenças do aparelho gastrointestinal, dentre outras. ${ }^{(3,11,12)}$

Os serviços de urgências e emergência atendem diariamente um fluxo intenso de usuários, cabendo aos profissionais médicos e enfermeiros o AcoIhimento adequado, identificando a clientela, neste nosso caso, o alcoolista, suprindo suas necessi- 
dades naquele momento e realizando encaminhamentos adequados, quando necessário. ${ }^{(13)}$

Dada a importância do Acolhimento aos usuários alcoolistas, questiona-se: Quais seriam as possíveis aplicações e contribuições do conceito de Acolhimento aos profissionais que atuam nos serviços de urgência e emergência frente ao cuidado prestado ao indivíduo alcoolista?. Com isso, este estudo tem por objetivo revisar a literatura acerca da aplicação do conceito de Acolhimento e suas contribuições frente à demanda específica do indivíduo alcoolista nestes serviços.

\section{MATERIAL E MÉTODOS}

Este estudo de abordagem qualitativa revisou a literatura para identificar a produção científica relacionada aplicação do conceito de Acolhimento e suas contribuições frente à demanda específica do indivíduo alcoolista nos serviços de urgência e emergência entre 2004 e 2014. Período no qual houve grande incentivo para a implantação da Política Nacional de Humanização de 2003, e em especial aos serviços de urgência e emergência, a formulação do documento norteador dos atendimentos no âmbito do SUS: Acolhimento com avaliação e classificação de risco, que pontua nomeadamente as ações voltadas ao processo de trabalho nos serviços de urgência e emergência em 2004. ${ }^{(14)}$

Optou-se pela realização de uma revisão integrativa, definida como um instrumento de obtenção, identificação, análise e síntese da literatura direcionada a um tema específico, que possibilita construir uma análise abrangente da literatura, debater sobre métodos e resultados alcançados pelas pesquisas já publicadas bem como uma associação entre o conhecimento teórico e prático, apontando evidências para a análise e soluções de problemas que ainda são pouco tratados pela literatura. ${ }^{15)}$

De acordo com critérios estabelecidos para a execução da pesquisa de revisão integrativa, foram seguidas seis etapas distintas e sequenciais: 1) elaboração da pergunta norteadora, ou seja, formula- ção do problema; 2) seleção da amostra, ou busca na literatura; 3) coleta de dados; 4) análise crítica dos estudos incluídos; 5) discussão dos resultados e 6) apresentação da revisão integrativa. ${ }^{(16)}$

O objeto de estudo foi a produção do conhecimento científico em periódicos acadêmicos sobre o Acolhimento e suas contribuições frente à demanda específica do indivíduo alcoolista nos serviços de urgência e emergência, nas seguintes bases de dados: Medical Literature and Retrieval System onLine (Medline), Literatura Latino-Americana e do Caribe em Ciências da Saúde (Lilacs), Base de Dados de Enfermagem (BDENF), e portal Scientific Electronic Library Online (SciELO). Os descritores utilizados tiveram como base a terminologia em saúde dos descritores em saúde (DeCS), da Biblioteca Virtual em Saúde, sendo eles: Acolhimento, emergência e álcool.

Para a elaboração da revisão integrativa, foram realizadas todas as tentativas possíveis combinando-se 3 descritores: Acolhimento AND Emergência AND Alcoolismo, porém em nenhuma delas foram encontrados resultados. Por isto, optou-se por realizar buscas somente com 2 descritores, sendo estes: Acolhimento AND alcoolismo; Acolhimento AND Emergência, e Emergência AND alcoolismo. A coleta de dados ocorreu entre os meses de janeiro a abril de 2015 .

Os critérios de inclusão estabelecidos para a seleção dos artigos foram: artigos publicados entre o período de janeiro de 2004 a dezembro de 2014, artigos originais de pesquisa de campo, nos seguintes idiomas: português, inglês ou espanhol, publicados em periódicos brasileiros e, estarem disponíveis na íntegra. Foram excluídos do estudo dissertações, teses, estudos de validação de instrumentos/escalas e livro texto, inclusive, foram excluídos os artigos que se repetiam entre as bases.

O processo de seleção dos estudos foi dividido em duas etapas. Na primeira, o refinamento ocorreu pela leitura analítica dos títulos e dos resumos. $\mathrm{Na}$ segunda, após a identificação dos artigos, foi feita uma exploração exaustiva das obras, isto é, 
leitura e releitura, sendo selecionadas aquelas que realmente atendiam aos critérios de inclusão.

A elaboração da revisão integrativa ocorreu conforme critérios e etapas estabelecidas na literatura. (16) Todas as etapas foram realizadas por dois pesquisadores independentes que realizaram a busca e leitura dos possíveis artigos que comporiam a revisão integrativa de acordo com os descritores de saúde selecionados. Quando houvesse discordância referente ao artigo selecionado por um dos pares para a inclusão do artigo, estes se reuniam e discutiam os pontos de discordância acatando ou não a inclusão do artigo na revisão integrativa baseando-se no objetivo do estudo, critérios de inclusão e conteúdo.

Visando à sistematização dos dados, foi utilizado um instrumento previamente validado na literatura. ${ }^{17)} \mathrm{Tal}$ instrumento permite avaliar separadamente cada artigo, por meio de um formulário que contempla os seguintes itens: identificação do artigo, características metodológicas do estudo, avaliação do rigor do método, intervenções mensuradas e resultados encontrados. Os artigos foram comparados e agrupados por similaridade de conteúdo, definindo-se duas categorias empíricas. ${ }^{(18)}$ Por fim, os artigos foram submetidos ao processo de extração de dados e, com base nisso, construído o texto científico.

\section{RESULTADOS}

Trata-se de um corpus de análise totalmente brasileiro, publicado em português, majoritariamente indexado na base de dados SciELO. A busca nas bases de dados identificou 137 estudos, dos quais 28 foram selecionados após aplicação dos critérios de inclusão, sendo que og apresentaram conteúdo relevante ao tema proposto, sendo 06 indexados na SciELO, o3 na LILACS e o (zero) na MEDLINE, conforme descrito na figura 1 .

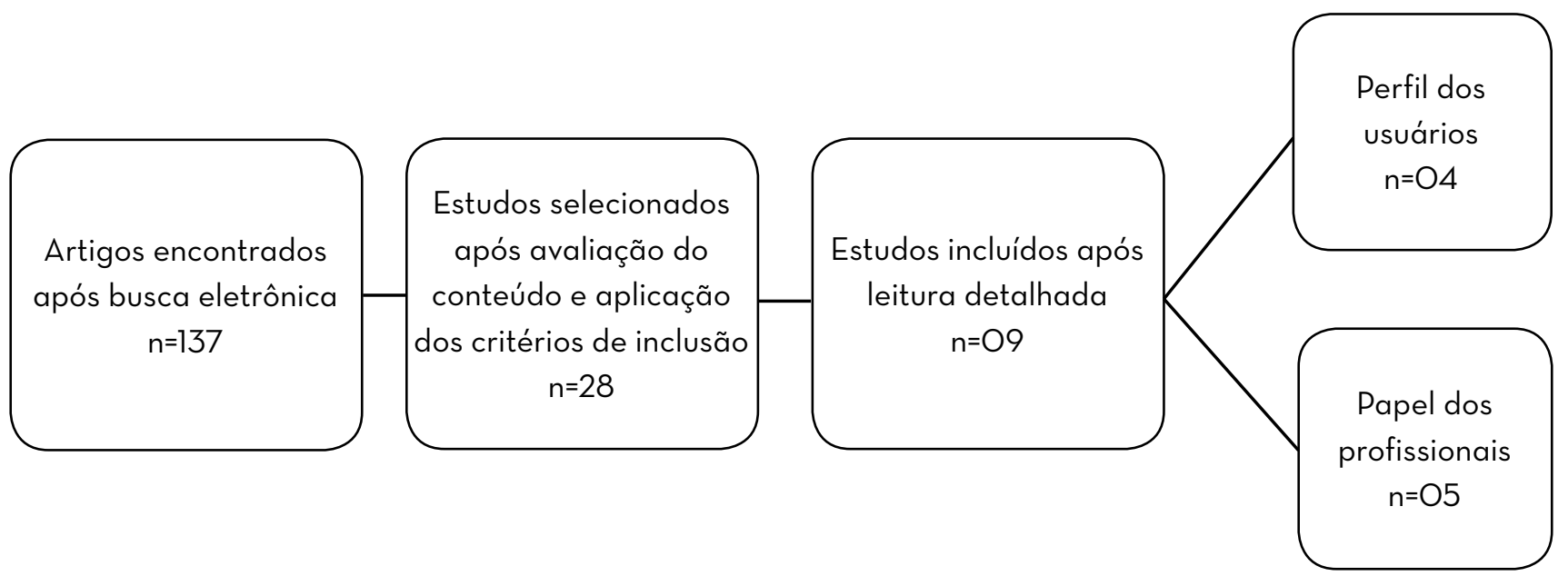

Figura 1 - Fluxograma com os resultados da busca eletrônica

Ao analisarem-se os resultados dos estudos, a fim de responder a questão de pesquisa desta revisão, revelou-se que a principal contribuição do conceito de Acolhimento frente à demanda específica do indivíduo alcoolista nos serviços de urgência e emergência emana das seguintes categorias: conhe- cimento do perfil do usuário dos serviços de urgência e emergência e o Acolhimento e o papel dos profissionais. Essas categorias encontram-se identificadas no quadro 1 e suas características principais descritas nos tópicos a seguir: 


\begin{tabular}{|c|c|c|c|}
\hline ANO & AUTORES & ARTIGO & CONTEÚdo \\
\hline 2006 & $\begin{array}{l}\text { MOTA; MARTINS; } \\
\text { VERAS }\end{array}$ & $\begin{array}{l}\text { Papel dos profissionais de saúde na política de } \\
\text { humanização hospitalar }\end{array}$ & $\begin{array}{l}\text { Papel dos } \\
\text { profissionais }\end{array}$ \\
\hline 2007 & $\begin{array}{l}\text { SOUZA; SILVA; } \\
\text { NORI }\end{array}$ & $\begin{array}{l}\text { Pronto-Socorro: uma visão sobre a interação entre } \\
\text { profissionais de enfermagem e pacientes }\end{array}$ & $\begin{array}{l}\text { Papel dos } \\
\text { profissionais }\end{array}$ \\
\hline 2007 & $\begin{array}{l}\text { SEGATTO; PINSKY; } \\
\text { LARANJEIRA; } \\
\text { REZENDE; VILELA. }\end{array}$ & $\begin{array}{c}\text { Triagem e intervenção breve em pacientes } \\
\text { alcoolizados atendidos na emergência: } \\
\text { perspectivas e desafios }\end{array}$ & $\begin{array}{l}\text { Papel dos } \\
\text { profissionais }\end{array}$ \\
\hline 2007 & MARQUES; LIMA & $\begin{array}{l}\text { Demandas de usuários a um serviço de pronto } \\
\text { atendimento e seu acolhimento ao sistema de saúde. }\end{array}$ & $\begin{array}{l}\text { Perfil dos } \\
\text { usuários }\end{array}$ \\
\hline 2010 & $\begin{array}{l}\text { SOUSA; SILVA; } \\
\text { OLIVEIRA }\end{array}$ & $\begin{array}{l}\text { Serviço de Emergência Psiquiátrica em hospital } \\
\text { geral: estudo retrospectivo }\end{array}$ & $\begin{array}{l}\text { Perfil dos } \\
\text { usuários }\end{array}$ \\
\hline 2011 & PRATTA; SANTOS & $\begin{array}{c}\text { O Processo Saúde-Doença e a Dependência } \\
\text { Química: Interfaces e Evolução }\end{array}$ & $\begin{array}{l}\text { Papel dos } \\
\text { profissionais }\end{array}$ \\
\hline 2011 & $\begin{array}{l}\text { BROSKA JUNIOR; } \\
\text { RIBAS; FURIATTI; } \\
\text { BOESE; PAIORNIK }\end{array}$ & $\begin{array}{c}\text { Perfil de atendimento de uma unidade de saúde } 24 \\
\text { horas na região sul de Curitiba }\end{array}$ & $\begin{array}{l}\text { Perfil dos } \\
\text { usuários }\end{array}$ \\
\hline 2011 & $\begin{array}{c}\text { OLIVEIRA; } \\
\text { SILVA; ARAUJO; } \\
\text { CARVALHO FILHO }\end{array}$ & $\begin{array}{l}\text { Perfil da população atendida em uma unidade de } \\
\text { emergência referenciada }\end{array}$ & $\begin{array}{l}\text { Perfil dos } \\
\text { usuários }\end{array}$ \\
\hline 2012 & $\begin{array}{l}\text { BONFADA; } \\
\text { GUIMARÃES }\end{array}$ & $\begin{array}{c}\text { Serviço de atendimento móvel de urgência e as } \\
\text { urgências psiquiátricas }\end{array}$ & $\begin{array}{l}\text { Papel dos } \\
\text { profissionais }\end{array}$ \\
\hline
\end{tabular}

Quadro 1 - Artigos analisados na Revisão Integrativa, segundo ano de publicação, autoria e categoria analítica que pertencem

\section{CONHECIMENTO DO PERFIL DOS USUÁRIOS DOS SERVIÇOS DE URGÊNCIA E EMERGÊNCIA}

Ainda vive-se uma grande procura por parte da clientela aos serviços de urgência e emergência, e isso deve-se, muitas vezes, à baixa qualidade e pouca oferta de vaga por agendamento nos serviços de saúde. ${ }^{(19)}$ A superlotação e o pouco contato pessoal não impedem a imensa procura por parte dos usuários do SUS, além de porta aberta, os serviços de urgência e emergência possuem um tempo de espera reduzida para a resolução dos problemas de saúde da população, seguindo a perspectiva curativa do processo saúde doença. ${ }^{(19)}$
A oferta restrita de serviços faz com que o público excedente procure atendimento em locais que concentrem maior possibilidade de portas de entrada, sendo que os pronto atendimentos e as emergências hospitalares correspondem ao perfil de atender às demandas de forma mais ágil e concentrada. Apesar de superlotados, impessoais e atuando sobre a queixa principal, esses locais reúnem um somatório de recursos, quais sejam consultas, remédios, procedimentos de enfermagem, exames laboratoriais e internações, enquanto as unidades de atenção básica oferecem apenas a consulta médica. ${ }^{(19)}$

A organização do SUS tem como uma de suas diretrizes a hierarquização, ficando sob competência dos serviços de urgência e emergência a resposta imediata das necessidades em saúde, atendendo a 
queixa principal. O Acolhimento deve estar presente em todas as formas de atendimento prestado pelos profissionais da saúde, incluindo os serviços de urgência e emergência, pois o Acolhimento implica-se na responsabilização de um atendimento com escuta qualificada, que oferte respostas adequadas aos usuários, encaminhando-os para uma assistência contígua. ${ }^{(19)}$

Em toda situação de atendimento prestado por profissionais de saúde, o acolhimento deve estar presente. Pressupõe atender a todos, ouvindo seus pedidos e assumindo uma postura capaz de dar respostas mais adequadas aos usuários, utilizando os recursos disponíveis para a resolução de problemas.

De acordo com a Política Nacional de Humanização (PNH) Humaniza SUS, o Acolhimento com avaliação e classificação de risco torna-se uma das intervenções potencialmente decisivas na reorganização e realização da promoção da saúde em rede. ${ }^{(20)}$

Conhecer as diferentes demandas presentes nos serviços de urgência e emergência faz-se necessário à oferta de prestação de serviços de qualidade às diferentes necessidades em saúde. Uma das necessidades específicas são os distúrbios psiquiátricos, tais são marcados por uma situação de crise, de desestabilidade, de ruptura, de perturbação, de conflitos, de desordem tanto em nível individual quanto coletivo. ${ }^{(21)}$ Estes podem ser conceituados como emergência: atendimentos que não podem ser protelados, devendo ser imediato; e urgências, em que $o$ atendimento pode ser prestado em tempo não superior a duas horas. ${ }^{(20)}$

Diante desse quadro, é indispensável a intervenção imediata de uma equipe multiprofissional, no intuito de evitar maiores prejuízos à saúde do indivíduo, ou de eliminar possíveis riscos à sua vida ou à de terceiros.

Embora tenha-se claro ambos conceitos de urgência e emergência, quando trata-se de distúrbios psiquiátricos estes podem não ser objetivos e por vezes mensurados, o que exige maior esforço dos profissionais de saúde, dado que indicadores objetivos por vezes não estão presentes nesses ca- sos. Essa iniciativa ainda é algo recente, visto que, o atendimento às urgências psiquiátricas no âmbito pré-hospitalar somente foi ratificado no Brasil pela primeira vez em 2004, garantindo uma articulação entre o SAMU e a Política Nacional de Saúde Mental. ${ }^{(22)}$

De acordo com um estudo realizado por Broska et al, ${ }^{(23)}$ dentre as 16 causas de maior procura pelo atendimento em unidades de urgência e emergência, a dependência alcóolica correspondeu a $(16,5 \%)$ dos atendimentos, destes, a maioria correspondeu ao sexo masculino $(83,6 \%)$ dos casos. Outro estudo relatado por Oliveira et al, ${ }^{(20)}$ aponta que o perfil da população atendida caracteriza-se por ser de adultos jovens, em idade produtiva, em dias úteis e em período diurno.

\footnotetext{
Como evidenciam os dados, houve um número significativo de atendimento de emergência psiquiátrica determinado pelo uso/abuso do álcool. Tal fato chama a atenção, pois o álcool é uma droga considerada de uso natural e é comum seu consumo no ambiente domiciliar, em festividades, ou mesmo em ambientes públicos. Neste aspecto, a sociedade é permissiva ao estimular referido consumo mediante propaganda.
}

Além disso, vale lembrar que, a demanda resultante do uso, abuso e dependência do álcool são recorrentes nos serviços de urgência e emergência, sejam eles em decorrência das causas externas que estes indivíduos se expõem ou ainda por complicações clínicas que necessitam de assistência de uma equipe de saúde. ${ }^{(24)}$

\section{O ACOLHIMENTO E O PAPEL DOS PROFISSIONAIS}

\begin{abstract}
A Psiquiatria foi criada por volta do século $X X$, tendo como objetivo a pesquisa de causas orgânicas provenientes de danos cerebrais que pudessem acarretar em patologias e agravos a saúde mental e com isso, surgiram uma grande quantidade de hospitais psiquiátricos que tinham em seus interiores muitos indivíduos rejeitados pela sociedade da época. ${ }^{(1)}$
\end{abstract}


No Brasil, desde a Reforma Psiquiátrica e com a Lei 10.216 promulgada em 2001, nota-se um movimento político e social unidos com objetivo da discussão de um novo conceito de atendimento e assistência para estes pacientes, que eram internados ou trancafiados nestes hospitais antes chamados de manicômios. ${ }^{(22)}$

O beber excessivo conduz claramente muitos pacientes à emergência com problemas de saúde física e mental. O profissional que atende esta demanda deve possuir a capacidade de identificar as pessoas cujo consumo de álcool pode prover risco à saúde, para que assim possa desenvolver um plano de intervenção. Uma das possíveis estratégias é a intervenção breve, com o intuito de motivar os indivíduos na mudança de seus hábitos. ${ }^{(24)}$

O principal objetivo da intervenção breve é reduzir o risco de danos ocasionados pelo consumo de substâncias psicoativas e com isso reduzir a possibilidade do aparecimento de problemas relacionados ao consumo de tais substâncias.

Segatto et al, (24) relatam a existência de algumas barreiras apontadas pelos profissionais da atenção primária, mas que também poderiam ser aplicados ao ambiente da urgência e emergência: falta de tempo, formação acadêmica insuficiente; medo de incomodar o paciente; crença de que os alcoolistas não respondem às intervenções.

Dentre outros problemas, o acesso aos serviços de saúde por parte dos indivíduos alcoolistas é comprometido, tanto por barreiras físicas quanto subjetivas. As barreiras subjetivas estão relacionadas ao estigma e/ou preconceito, isso porque, os cuidados aos alcoolistas no Brasil ainda está muito relacionado a modelos punitivos de tratamento e de reclusão. ${ }^{(25)}$ Com isso, o processo de trabalho dos profissionais de saúde que prestam atendimento nesses serviços, deve convergir às mudanças e adaptações que resgatem a humanização do cuidado, compartilhando com seu paciente as experiências e vivências a fim de ampliar o foco de suas ações. ${ }^{(13)}$
Junto aos desafios no atendimento em emergência o saber ouvir torna-se primordial para a realização do Acolhimento, bem como, poder atender as expectativas dos pacientes. Por outro lado, o discurso presente ainda é a mecanização do cuidado, pois o cuidar ainda resume-se aos procedimentos realizados. ${ }^{(26)}$

\footnotetext{
Dar atendimento humanizado não requer, necessariamente, dedicar mais tempo ao paciente ou ter condições de trabalho ideais. Basta, para tanto, saber dirigir palavras de conforto e segurança, dar real atenção à pessoa, conversar com ela, deixar que se manifeste e, até, que reclame.
}

Os profissionais de saúde devem dispor de condições necessárias para desenvolver suas atividades, lembrando que o tratamento humanizado não se concretiza se estiver centrado no paciente, com isso, a conscientização das especificidades das diferentes demandas, poderá ser alcançada com meIhorias na comunicação e ruptura com o modelo que privilegia a mera rotina de procedimentos. ${ }^{(26)}$

Uma importante contribuição foi dada ao papel da equipe de enfermagem, pois esta deve estar preparada para promover ações de transformação, visando não somente a promoção ao cuidado individual à saúde, agindo também em prol da transformação social, e muitas vezes visando à reinserção social em busca de mais qualidade na assistência prestada. ${ }^{(13)}$

\section{DISCUSSÃO}

$\mathrm{Na}$ leitura dos artigos observou-se que uma porcentagem expressiva de trabalhos que apresentam - Acolhimento em saúde mental e ao usuário alcoolista referem-se ao âmbito da Atenção Básica e também aos atendimentos especializados, como é o caso do CAPS (Centro de Atenção Psicossocial), poucas foram as obras que tratam do indivíduo alcoolista em unidades de urgência e emergência. Por esta razão, muitos trabalhos foram excluídos na etapa 02 da revisão: seleção da amostra. 
Nos estudos em que o contexto hospitalar era exposto, notou-se uma ênfase aos institutos de psiquiatria, ignorando a demanda presente em inúmeros serviços de urgências e emergência que tratam de indivíduos alcoolistas, nas suas diferentes demandas, sejam elas por: intoxicação aguda, distúrbios metabólicos, acidentes e violências; e que necessitam de cuidado humanizado e acolhedor.

Atualmente os profissionais da saúde necessitam realizar adaptações que requerem habilidades e conhecimentos específicos necessários ao atendimento dos usuários de álcool e drogas, exigindo uma mudança na capacidade de trabalho em grupo, facilitando a adaptação aos novos ambientes de trabalho e clientela de forma a atender suas necessidades e expectativas de resolução de problemas de saúde. . $^{(6,27)}$

Por outro lado, os profissionais que atuam no serviço de emergência sofrem, pois trabalham contra o tempo, sob pressão, necessitando de uma assistência precisa, que intervenha da melhor maneira, superando situações que retratam a saúde em nosso país. ${ }^{\left({ }^{10}\right)} A$ equipe de enfermagem apresenta-se como importante e essencial nos cuidados da população nos serviços de urgências e emergência, mas que se tratando da assistência a pacientes alcoolistas, esses cuidados são vistos pelos profissionais de maneira a questionar a forma correta de treinamento profissional, ambiente de trabalho seguro e até mesmo a real importância do indivíduo estar naquele serviço de saúde. ${ }^{(28)}$

Relacionado a isso, encontra-se um desgaste psicoemocional nos profissionais, que, embora a técnica exista para auxiliar nos cuidados prestados aos pacientes, o fluxo intenso de usuários, a falta de informação dos mesmos, a jornada de trabalho exaustiva proporciona limitações de dedicação e vínculo ao paciente. ${ }^{(29)}$

Sendo a enfermagem uma categoria profissional apontada com grande importância tanto no Programa Nacional de Humanização como também, na articulação do Acolhimento nos serviços de urgência e emergência, pois o enfermeiro é protagonista no Sistema de Classificação de Risco na porta de entrada dos serviços, uma lacuna importante a ser desvelada é a formação dos enfermeiros no país. Pouca atenção vem sendo dada à questão do álcool nos currículos de graduação em enfermagem no Brasil, e o reflexo deste acontecimento tem sido a dificuldade de aceitar o conceito de alcoolismo como doença, associado a atitudes e ideais preconceituosos e negativos e levando à falta de preparo para lidar com esta demanda. ${ }^{(30)}$

\section{CONSIDERAÇÕES FINAIS}

Foi possível identificar a inserção do conceito de Acolhimento nas práticas de urgência e emergência, porém isso pouco relacionado às especificidades do indivíduo alcoolista, embora ele seja uma demanda frequente nos serviços.

Muitas mudanças sociais e culturais ocorreram com a implantação da humanização nos serviços de saúde, mas o paciente alcoolista ainda enfrenta o preconceito, discriminação, abandono por parte dos familiares e a visão de sua doença ligada a falta de caráter e ausência de protocolos de cuidados adequados, prejudicam o Acolhimento de forma integral, porém, não se pretende esgotar o assunto, mas estimular o diálogo com pesquisas vindouras.

Conclui-se que a utilização das premissas do AcoIhimento em Saúde no atendimento aos indivíduos alcoolistas, em especial nos serviços de urgência e emergência, deve-se basear-se em duas características importantes: primeiramente o reconhecimento das especificidades e necessidades próprias aos diferentes perfis de usuários presentes nos serviços e, posteriormente, o delineamento e a clareza do AcoIhimento, para que os profissionais entendam-se como agentes transformadores da realidade, e que possam atender as recomendações constantes na Política Nacional de Humanização, nos diferentes níveis de atenção à saúde. 


\section{REFERÊNCIAS}

1. Pratta EMM, Santos MA dos. O processo saúde-doença e a dependência química: interfaces e evolução. Psic Teor Pesqui. 2009[acesso em 2015 dez 21];45(2):203211. Disponível em: http://dx.doi.org/10.1590/ SO1O2-37722009000200008

2. Marques ACPR. O uso do álcool e a evolução do conceito de dependência de álcool e outras drogas e tratamento. Revista IMESC. 2001;(3):73-86.

3. Sordi AO, Kreische F, Diemen von L. Álcool: tratamento de dependência de crack, álcool e outras drogas. In: Ministério da Justiça (BR). Secretaria Nacional de Políticas Sobre Drogas. Tratamento da dependência de crack, álcool e outras drogas: aperfeiçoamento para profissionais de saúde e assistência social. Brasília: SENAD; 2013.

4. Gonçalves JRL, Galera SAP. Assistência ao familiar cuidador em convívio com alcoolista, por meio da técnica de solução de problemas. Rev Lat Am Enfermagem. 2010 [acesso em 2015 dez 21];18(Spec):543-549. Disponível em: http://dx.doi.org/10.159O/SO1O411692010000700009

5. Botti NCL, Lima AFD, Simões WMB. Uso de substâncias psicoativas entre acadêmicos de enfermagem da universidade católica de Minas Gerais. SMAD, Rev Eletrônica Saúde Mental Alcool Drog. 2010 [acesso em 2015 dez 21];6(1):1-13. Disponível em: http://dx.doi. org/10.11606/issn.1806-6976.v6ilpl-20

6. Oliveira C, Vargas, D. Representações sociais dos enfermeiros de hospital geral diante do paciente alcoolista. Cogitare enferm. 2012 [acesso em $2015 \mathrm{dez} 21]$;17(3):452-7. Disponível em: http://dx.doi.org/10.5380/ce.v17i3.24644

7. Tesser CD, Neto PP, Campos GW. Acolhimento e (Des)medicalização social: um desafio para as equipes de saúde da família. Ciênc Saúde Coletiva. 2010;15(supl 3):3615-3624.

8. Carvalho DOC, Santos NNRC, Silva ARV, Carvalho GCN. Percepção do profissional de enfermagem acerca do cuidado humanizado no ambiente hospitalar. Rev Interdisciplin. 2015;8(3):61-74.
9. Mota RA, Martins CG, Véras RM. Papel dos profissionais de saúde na Política de Humanização Hospitalar. Psicol. estud. 2006 [acesso $2015 \mathrm{dez} 21$ ];11(2):323-330. Disponível em: http://dx.doi.org/10.1590/S141373722006000200011

10. Pai DD, Lautert L. Suporte humanizado no Pronto Socorro: um desafio para a enfermagem. Rev Bras Enferm. 2005 [acesso em 2015 dez 21]; 58(2):231-234. Disponível em: http://dx.doi. org/10.159O/SO034-71672005000200021

11. Strauch E, Schneider PRT, Silva RA, Horta BL. Uso de álcool por adolescente: Estudo de base populacional. Rev Saúde Pública. 2009 [acesso em 2015 dez 21];43(4):647-655. Disponível em: http://dx.doi.org/10.1590/SO03489102009005000044

12. Portugal FB, Correa APM, Siqueira MM. Alcoolismo e comorbidade em um programa de assistência aos dependentes de álcool. SMAD, Rev Eletrônica Saúde Mental Alcool Drog. 2010;(6):1-13.

13. Gonçalves SSPM, Tavares CMM. Atuação do enfermeiro na atenção ao usuário de álcool e outras drogas nos serviços extra hospitalares. Esc Anna Nery Rev Enferm. 2007 [acesso em 2015 dez 21];11(4):586-592. Disponível em: http://dx.doi.org/10.5216/ree.v14i2.11245

14. Ministério da Saúde (BR). Secretaria de Atenção à Saúde. Política Nacional de Humanização da Saúde. Gestão participativa e cogestão. Brasília, DF; 2009.

15. Ganong LH. Integrative reviews of nursing research. Res Nurs Health. 1987;10(1):1-11.

16. Felix TA, Ferreira FV, Oliveira EM, Eloia SC, Gomes BV, Eloia SMC. Prática da humanização na visita em uma unidade de terapia intensiva. Revista Enfermagem Contemporânea 2014 [acesso em 2015 dez 21];3(2):143-153. Disponível em: http://dx.doi.org/10.17267/2317-3378rec. v3i2.381

17. Ursi ES, Gavão CM. Prevenção de lesões de pele no perioperatório: revisão integrativa da literatura. Rev Lat Am Enfermagem. 2014 [acesso em 2015 dez 21];14(1):124-131. Disponível em: http://dx.doi.org/10.1590/SO10411692006000100017 
18. Garuzi M, Achitti MCO, Sato CA, Rocha SA, Spagnuolo RS. Acolhimento na Estratégia Saúde da Família: revisão integrativa. Rev Panam Salud Publica. 2014;35(2):144-149.

19. Marques GQ, Lima MA. Demandas de usuários a um serviço de pronto atendimento e seu acolhimento ao sistema de saúde. Rev Lat Am Enfermagem. 2007 [acesso em $2015 \mathrm{dez}$ 21];15(1):O1-O8. Disponível em: http://dx.doi. org/10.1590/SO104-11692007000100003

2O. Oliveira GN, Silva MFN, Araujo IEM, Filho MA. Perfil da população atendida em uma unidade de emergência referenciada. Rev Lat Am Enfermagem. 2011;19(3):1-9.

21. Sousa FSP, Silva CAF, Oliveira EN. Serviço de Emergência Psiquiátrica em hospital geral: estudo retrospectivo. Rev Esc Enferm USP. 2010 [acesso em 2015 dez 21];44(3):796-802. Disponível em: http://dx.doi.org/10.1590/ S0080-62342010000300035

22. Bonfada D, Guimaraes J. Serviço de atendimento móvel de urgência e as urgências psiquiátricas. Psicol Estud Maringá. 2012;17(2):227-236.

23. Broska J, Ribas CA, Furiatti EO, Baggio G, Boese LCR, Paciornik EF. Perfil de atendimento de uma unidade de saúde 24 horas na região sul de Curitiba. Revista Eletrônica da Faculdade Evangélica do Paraná, Curitiba. 2011;1(2):31-40.

24. Segatto ML, Pinsky I, Laranjeira R, Rezende FF, Vilela TR. Triagem e intervenção breve em pacientes alcoolizados atendidos na emergência: perspectivas e desafios. Cad Saúde Pública. 2007;23(8):1753-1762.
25. Quinderé PHD. A experiência do uso do crack e sua interlocução com a clínica: dispositivos para o cuidado integral do usuário [tese]. Ceará: Universidade do Ceará; 2013.

26. Souza RB, Silva MJP, Nori A. Pronto-Socorro: uma visão sobre a interação entre profissionais de enfermagem e pacientes. Rev Gaúch Enferm. 2007;28(2): 242-9.

27. Lima LPM, Santos AAP, Póvoa FTX, Silva FCL. O papel do enfermeiro durante a consulta de pré-natal à gestante usuária de drogas. Revista Espaço para a Saúde. 2015; 16(3):39-46.

28. Campos CJG, Teixeira MB. O atendimento do doente mental em pronto-socorro geral: sentimentos e ações dos membros da equipe de enfermagem. Rev Esc Enferm USP. 2001 [acesso em 2015 dez 21]; 35(2):141-9. Disponível em: http://dx.doi.org/10.1590/SO08O62342001000200008

29. Elias MA, Navarro VL. A relação entre o trabalho, a saúde e as condições de vida: negatividade e positividade no trabalho das orofisssionais de enfermagem de um hospital escola. Rev Lat Am Enfermagem. 2006 [acesso em 2015 dez 21];14(4):517-525. Disponível em: http://dx.doi.org/10.1590/SO10411692006000400008

30. Vargas D. Atitudes de estudantes de enfermagem frente questões relacionadas ao álcool, alcoolismo e alcoolista. Acta Paul Enferm. 2011 [acesso em $2015 \mathrm{dez}$ 21];24(5):638-644. Disponível em: http://dx.doi. org/10.1590/SO103-21002011000500007 